\title{
Relation between adverse events associated with allopurinol and renal function in patients with gout
}

\author{
J Vázquez-Mellado, E Meoño Morales, C Pacheco-Tena, R Burgos-Vargas
}

\begin{abstract}
Background-Because serious adverse reactions to allopurinol have been related to a reduce creatinine clearance rate and prolonged half life of oxypurinol, it has been recommended that the dose should be adjusted according to the rate of creatinine clearance. However, in some patients with gout the dose is not sufficient to reduce serum levels of uric acid $(\leqslant 390 \mu \mathrm{mol} / \mathrm{l})$ and to halt disease progression.
\end{abstract}

Objective-To determine the prevalence of adverse reactions attributable to allopurinol in patients with primary gout according to dose and creatinine clearance rate.

Methods-Data on 120 patients with gout receiving allopurinol, in whom the starting dose was adjusted according to creatinine clearance rate and later increased in some patients to control the disease, were retrospectively reviewed. Two groups were compared: group $\mathrm{A}, 52$ patients receiving creatinine clearance adjusted maintenance doses of allopurinol and group $B, 68$ patients receiving non-adjusted higher maintenance doses of allopurinol.

Results-During follow up $\mathbf{5 7 \%}$ required higher allopurinol doses than those recommended according to their creatinine clearance rate. Only five $(4 \%)$ of 120 consecutive patients developed allopurinol related adverse reactions: four minor skin reactions and one allopurinol hypersensitivity syndrome (AHS). Three of these (including the case of AHS) occurred in group $A$ and two in group $B$ $(p=N S)$. The duration of allopurinol treatment was the same in both groups (group A: 2.3 (3.3) years; group B: 3.7 (4.8) years). No patient in group $A$, but $44 \%$ in group $B$ had a creatinine clearance rate of $<50 \mathrm{ml} /$ min. None of the patients received concomitant diuretics, ampicillin, or azathioprine.

Conclusions-No increase was seen in the prevalence of adverse reactions to allopurinol in patients who received higher allopurinol maintenance doses than those recommended according to creatinine clearance rate.

(Ann Rheum Dis 2001;60:981-983)

Allopurinol is the urate lowering drug most frequently used in patients with gout and other hyperuricaemic conditions. It has been estimated that approximately $2 \%$ of patients treated with allopurinol develop minor adverse reactions which often disappear after stopping the drug. Serious and life threatening adverse events such as the allopurinol hypersensitivity syndrome (AHS) are rare. ${ }^{1-5}$ Although most adverse reactions to allopurinol probably result from individual idiosyncrasies, ${ }^{6}$ Hande et al suggested a direct relation between severe allopurinol toxicity and decreased creatinine clearance $^{7}$ and proposed adjusting the dose of allopurinol according to the rate of creatinine clearance to reduce the risk of severe toxicity. Since then it has been common practice to follow this recommendation in treating patients with hyperuricaemia or gout and a decreased creatinine clearance rate. ${ }^{18-11}$

One of the most important objectives in treating patients with gout is to reduce the serum level of uric acid and to halt the episodes of arthritis, tophi growth, joint deformities, and radiographic changes. This goal is usually achieved by reducing the serum concentration of uric acid to $390 \mu \mathrm{mol} / 1$ by giving allopurinol in daily doses of $300 \mathrm{mg}$ (range 100$800 \mathrm{mg}) .{ }^{8911}$ Unfortunately, some patients with gout who receive allopurinol in doses adjusted to the creatinine clearance rate may not benefit from the use of lower doses of the drug, and higher doses are needed to reduce the serum uric acid level. Increased doses in such cases do not appear to be linked to a higher prevalence of serious adverse reactions. We have investigated the prevalence of adverse events attributable to allopurinol in patients with gout and its relation with the creatinine clearance rate.

\section{Methods}

A retrospective study was performed in which the clinical records of consecutive patients with gout receiving regular treatment with allopurinol for at least 1 month before their inclusion in the analysis were reviewed. In all patients the initial dose of allopurinol was adjusted according to the creatinine clearance rate. During the follow up period the dose of allopurinol had to be increased in some patients to achieve a sustained reduction in uric acid levels. In these patients the daily dose of allopurinol exceeded the dose adjusted for the creatinine clearance rate.

Study variables included demographic and clinical data, associated diseases and their treatment, as well as details on allopurinol treatment such as daily and accumulated doses 
Table 1 Major associated conditions, significant laboratory data, and treatment in patients with gout receiving allopurinol

\begin{tabular}{|c|c|c|c|c|c|}
\hline \multirow[b]{2}{*}{ Diabetes mellitus (n, \%) } & \multicolumn{2}{|c|}{ Group $A$ n=52 } & \multicolumn{2}{|c|}{ Group B $n=68$} & \multirow{2}{*}{$\frac{p \text { Value }}{\text { NS }}$} \\
\hline & 3 & (5.7) & 7 & $(10.2)$ & \\
\hline Hypertension (n, \%) & 13 & (25) & 20 & (29) & NS \\
\hline Hyperlipidaemia (n, \%) & 13 & (25) & 12 & (18) & NS \\
\hline Haemoglobin $(\mathrm{g} / \mathrm{l})^{\star}$ & 160 & (13) & 146 & (26) & 0.002 \\
\hline Packed cell volume & 0.49 & $(0.04)$ & 0.46 & $(0.08)$ & 0.005 \\
\hline Creatinine $(\mu \mathrm{mol} / 1)^{\star}$ & 100 & $(18)$ & 120 & $(53)$ & 0.003 \\
\hline $\mathrm{BUN}(\mathrm{mmol} / \mathrm{l})^{\star}$ & 11.5 & $(4.5)$ & 16.5 & $(8.0)$ & 0.000 \\
\hline $\mathrm{CrC}(\mathrm{ml} / \mathrm{min})^{\star}$ & 103.8 & $(21.8)[99.7]$ & 54.6 & $(20.1)[52.9]$ & 0.000 \\
\hline \multicolumn{6}{|c|}{ Allopurinol maintenance doses (mg/day) } \\
\hline $100-<300, \mathrm{n}(\%)$ & 4 & (8) & 1 & (2) & NS \\
\hline $300, \mathrm{n}(\%)$ & 46 & (88) & 62 & (91) & NS \\
\hline$>300, \mathrm{n}(\%)$ & 2 & (4) & 5 & (7) & NS \\
\hline Other treatments, $\mathrm{n}(\%)$ & 34 & (65) & 49 & (72) & NS \\
\hline
\end{tabular}

BUN = blood urea nitrogen; $\mathrm{CrC}=$ creatinine clearance.

*Values represent mean (SD) [median].

and duration of treatment. Laboratory data included complete blood cell count, blood chemistry, urine analysis, and creatinine clearance rate normalised for a body surface area of $1.73 \mathrm{~m}^{2}$ in 24 hour urine samples. Particular attention was paid to past and present clinical or laboratory adverse events and their relation with allopurinol.

Adverse events were defined as all new clinical and laboratory data not explained by gout or concomitant diseases that appeared during follow up. Those requiring admission to hospital or producing permanent incapacity were considered serious; all others were classified as non-serious adverse events.

For the analysis the patients were divided into two groups. Group A comprised patients whose allopurinol maintenance dose matched the dose schedule recommended by Hande et $a l^{7}$ according to their creatinine clearance rate $(0 \mathrm{ml} / \mathrm{min}, 100 \mathrm{mg}$ every 3 days; $10 \mathrm{ml} / \mathrm{min}$, $100 \mathrm{mg}$ every 2 days; $20 \mathrm{ml} / \mathrm{min}, 100 \mathrm{mg}$ daily; $40 \mathrm{ml} / \mathrm{min}, 150 \mathrm{mg} /$ day; $60 \mathrm{ml} / \mathrm{min}, 200 \mathrm{mg} /$ day; $80 \mathrm{ml} / \mathrm{min}, 250 \mathrm{mg} /$ day; $100 \mathrm{ml} / \mathrm{min}$, $300 \mathrm{mg} /$ day; $120 \mathrm{ml} / \mathrm{min}, 350 \mathrm{mg} /$ day; $140 \mathrm{ml} /$ $\mathrm{min}, 400 \mathrm{mg} /$ day). Group B comprised patients whose maintenance dose exceeded the dose recommended for their creatinine clearance rate. Comparisons between groups were carried out using the $\chi^{2}$ test, Fisher's exact test, and $t$ test.

\section{Results}

One hundred and twenty consecutive patients with primary gout (118 men, two women) were included in the analysis. Their mean (SD) age was 52.7 (12.4) years (median 55) and disease duration was 13.4 (8.1) years (median 12). Allopurinol had been administered for a mean of 3.3 (4.5) years (median 1.2) in daily doses ranging from $100 \mathrm{mg}$ to $450 \mathrm{mg}$ (table 1). Concomitant diseases were: hypertension in 33 (54\%), hyperlipidaemia in 25 (43\%), and diabetes mellitus in 10 (16\%). Renal failure, as defined by a creatinine clearance rate of $50 \mathrm{ml} /$ min or less, was diagnosed in 30 patients (44\%).

Only five patients ( $4 \%$, all men) from the whole group developed allopurinol related adverse reactions (table 2). Four patients had minor reactions within 5-32 months of starting treatment, consisting of a skin rash in two cases (both in group A), mild cutaneous vasculitis in one (group B), and fixed pigmented drug eruption in one case (group B). The only serious adverse event was AHS which was diagnosed within 2 weeks of starting allopurinol treatment in a 36 year old man in group A with a 16 year history of tophaceous gout and a normal creatinine clearance rate (reported elsewhere). ${ }^{12}$ In all patients, including the latter after drug desensitisation, allopurinol rechallenge with escalating doses did not result in any adverse reactions. Thus, three adverse reactions, including one serious event, occurred in group A and two in group B (5.8\% versus $3.0 \%, \mathrm{p}=\mathrm{NS}$ ).

Most variables were further analysed by group. Group A comprised 52 patients (all men) and group B consisted of 68 patients (66 men and two women). The mean age and duration of disease in group A was significantly lower than in group B (48.3 (10.9) years $v 56.2$ (12.1) years, $\mathrm{p}=0.002$; and 11.7 (6.4) years $v$ 15 (9.0) years, $p=0.05)$. Serum uric acid levels did not differ between groups during the follow up period (group A: 400 (314) $\mu \mathrm{mol} / 1$ (median 390); group B: 390 (172) $\mu \mathrm{mol} / \mathrm{l}$ (median 360), $\mathrm{p}=\mathrm{NS}$ ). The mean duration of treatment with allopurinol was similar at the time of the analysis (2.3 (3.3) years in group A, 3.7 (4.8) years in group B). No patient in group A but 30 in group B $(44 \%)$ had renal failure. The prevalence of hyperlipidaemia, hypertension, and diabetes mellitus in the two groups was similar (table 1), although there were several differences in laboratory test results between the two groups. Thirty four patients in group A $(65 \%)$

Table 2 Adverse effects associated with allopurinol

\begin{tabular}{|c|c|c|c|c|c|}
\hline & Patient 1 & Patient 2 & Patient 3 & Patient 4 & Patient 5 \\
\hline Group & A & A & A & B & B \\
\hline Age (years) & 56 & 66 & 36 & 56 & 60 \\
\hline $\begin{array}{l}\text { Duration of the disease } \\
\text { (years) }\end{array}$ & 12 & 23 & 16 & 29 & 3.3 \\
\hline $\begin{array}{l}\text { Previous duration of } \\
\text { treatment (months) }\end{array}$ & 5 & 32 & 0.5 & 6 & 18 \\
\hline $\begin{array}{l}\text { Allopurinol maintenance } \\
\text { doses (mg/day) }\end{array}$ & 300 & 300 & 300 & 300 & 300 \\
\hline $\mathrm{CrC}(\mathrm{ml} / \mathrm{min})$ & 115.8 & 92 & 108 & 80 & 61.2 \\
\hline Adverse event & Rash & Rash & AHS & FPE & $\begin{array}{l}\text { Leucocytoclastic } \\
\text { vasculitis }\end{array}$ \\
\hline Subsequent ULD & $\begin{array}{l}\text { Allopurinol } \\
\text { reinitiated }\end{array}$ & $\begin{array}{l}\text { Allopurinol } \\
\text { reinitiated }\end{array}$ & $\begin{array}{l}\text { Allopurinol } \\
\text { reinitiated }^{\star}\end{array}$ & $\begin{array}{l}\text { Allopurinol } \\
\text { reinitiated }\end{array}$ & $\begin{array}{l}\text { Allopurinol } \\
\text { reinitiated }\end{array}$ \\
\hline
\end{tabular}

$\mathrm{CrC}=$ creatinine clearance; $\mathrm{AHS}=$ allopurinol hypersensitivity syndrome; FPE $=$ fixed pigmented drug eruption; ULD = urate lowering drug.

*Allopurinol was reinitiated after the patient received benzobromarone for 6 years without adequate response and after desensitisation, as described in Results section. ${ }^{17}$ 
and 49 in group B (72\%) received additional treatment, mainly antihypertensive drugs (angiotensin converting enzyme inhibitors or calcium blockers) and non-steroidal antiinflammatory drugs, but none received diuretics, ampicillin, or azathioprine.

\section{Discussion}

The results of this study show that, although more than half the patients were treated with allopurinol in doses higher than those corresponding to their creatinine clearance rate, the frequency of adverse events was very low and similar to that found in patients given doses adjusted to the creatinine clearance rate. ${ }^{28}$ This finding may be relevant when treating populations with gout in which there is a high prevalence of renal failure. Differences from previous reports may be related to race, age, concomitant medications and morbidity, and also to study design. Most previous reports have described individual cases or series of patients with adverse events related to allopurinol but, interestingly, only a minority had gout $^{1-7}$ and patients receiving allopurinol who did not develop adverse events were not included. There is only one study reporting allopurinol associated adverse reactions in hospitalised patients with comorbid conditions and receiving multiple treatments. ${ }^{5}$

The proposal by Hande et al was based on six of their patients and 72 cases with severe allopurinol toxicity reported in the literature. Only $44 \%$ of these patients had a clear indication for allopurinol; $81 \%$ in whom data were available had renal failure before starting allopurinol; $49 \%$ were receiving concomitant diuretic treatment; and serious adverse events associated with allopurinol appeared within 3 weeks of treatment. Hande et al showed that the renal clearance of oxypurinol was directly proportional to the creatinine clearance rate and attributed the appearance of severe toxicity to this finding. They therefore proposed guidelines to dose adjustment and these are widely recommended. ${ }^{811}$

Although the retrospective nature of our study limits its scope, our findings challenge the role of the prolonged half life and raised serum concentrations of oxypurinol in the pathogenesis of serious adverse reactions. This issue should be further investigated and tested against a number of other factors implicated in the pathogenesis of serious toxicity. ${ }^{73}$

Whether physicians follow any guideline for adjusting the dose of allopurinol according to the creatinine clearance rate is unknown. In the 1980s more than five million patient years of treatment were accumulated and 240 million doses of allopurinol were prescribed or 70 tonnes ingested each year for a variety of conditions. ${ }^{2}$ We believe that a significant proportion of patients with a low creatinine clearance rate seen in general practice receive nonadjusted doses of allopurinol, but severe toxicity is rare. In contrast, minor adverse reactions to allopurinol probably occur in $1.8-2.0 \%$ of patients receiving the drug. ${ }^{2514}$ The cause of severe toxicity to allopurinol, including AHS, is unknown. Hypersensitivity, genetic predisposition, and a number of factors such as age, concomitant diseases, and medications like ampicillin and diuretics are likely to play a role in its pathogenesis. $^{21415}$

One recent report ${ }^{16}$ suggests that benzobromarone could be effective as a urate lowering drug in patients with gout, decreasing excretion of uric acid, and renal impairment but, unfortunately, it is not yet available in all countries.

In conclusion, from the results of our study we suggest the following recommendations for allopurinol treatment: (1) Verify that there is a clear indication for allopurinol treatment. (2) Start allopurinol in doses according to the creatinine clearance rate. (3) Check for concomitant diseases or treatment associated with serious adverse events in patients requiring higher doses of allopurinol. (4) In patients for whom no other urate lowering drug is available, the dose of allopurinol should be increased with caution.

1 Cameron JS, Simmonds HA. Use and abuse of allopurinol. BMJ 1989;294:1504-5.

2 Arellano F, Sacristán J. Allopurinol hypersensitivity syndrome: a review. Ann Pharmacother 1993;27:337-43.

3 Tam S, Carroll W. Allopurinol hepatotoxicity. Am J Med 1989;86:357-8

4 Auböck J, Fritsch P. Asymptomatic hyperuricemia and allopurinol induced toxic epidermal necrolysis. BMJ 1985; 290:1969-70.

5 McInnes GT, Lawson DH, Jick H. Acute adverse reactions attributed to allopurinol in hospitalised patients. Ann Rheum Dis 1981;40:245-9.

6 Emmerson BT, Hazelton RA, Frazer IH. Some adverse reactions to allopurinol may be mediated by lymphocyte reactivity to oxypurinol. Arthritis Rheum 1988;31:436-40.

7 Hande KR, Noone RM, Stone WJ. Severe allopurinol toxicity. Am J Med 1984;76:47-56.

8 Emmerson BT. The management of gout. N Engl J Med 1996;334:445-51.

9 Fam AG. "Hyperuricemia and gout". In: Rakel RE, ed. Conn's current therapy. 51 st edn. Philadelphia: Saunders, 1999:564-5.

10 Huey WY, Coyne DW. Dosage adjustments of drugs in renal failure. In: Washington manual of medical therapeutics. 29th edn. New York: Lippincott-Raven, 1998:549-55

11 Wortmann RL. Management of hyperuricemia. In: Koopman WJ, ed. Arthritis and allied conditions. 13th edn. Baltimore: Williams \& Wilkins, 1997:2073.

12 Vázquez-Mellado J, Guzmán $\mathrm{S}$, Cazarín Barrientos J, Gómez Ríos V, Burgos Vargas R. Desensitization to allopurinol after allopurinol hypersensitivity syndrome with renal involvement in gout. Case report. J Clin Rheumatol 2000; involvement

13 Hande KR. Evaluation of a thiazide-allopurinol drug interaction. Am J Med Sci 1986;292:213-6.

14 Singer JZ, Wallace SL. The allopurinol hypersensitivity syndrome. Arthritis Rheum 1986;29:82-7.

15 Fam AG, Lewtas J, Stein J, Paton TW. Desensitisation to allopurinol in- patients with gout and cutaneous reactions. Am J Med 1992;93:299-302.

16 Pérez Ruiz F, Alonso Ruiz A, Calabozo M, Herrero-Beites A, García-Erauskin G, Ruiz-Licea E. Efficacy of allopurinol and benzobromarone for the control of hyperuricemia. A pathogenic approach to the treatment of primary chronic gout. Ann Rheum Dis 1998;57:545-9. 